\title{
TESTS OF A POLARIZED SOURCE OF HYDROGEN AND DEUTERIUM BASED ON SPIN-EXCHANGE OPTICAL PUMPING AND A STORAGE CELL FOR POLARIZED DEUTERIUM
}

k. J. Holt, R. Gilman, E. R. Kinney, R. S. Kowalczyk, J. Napolitano, L. Young

Argonne National Laboratory, Argonne, IL 60439-4843 USA

S. I. Mishinev, D. M. Nikolenko, S. G. Popov, I. A. Rachek,

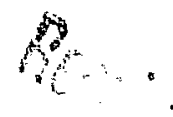

A. B. Temnykh, D. K. Toporkov, E. P. Tsentalovich,

D. K. Vesnorsky, B. B. Wojtsekhowski, V. G. Zelevinsky

Institute of Nuclear Physics, Novosibirsk, 630090, USSR

\begin{abstract}
A novel laser-driven polarized source of hydrogen and deuterium which is based on the principle of spin-exchange optical pumping has been developed at Argonne. The advantages of this method over conventional polarized sources for internal target experiments is discussed. At present, the laser-driven polarized source delivers hydrogen $8 \times 10^{16}$ atoms/s with a polarization of $24 \%$ and deuterium at $6 \times 10^{18}$ atoms/s with a polarization of $25 \%$. A passive storage cell for polarized deuterium was tested in the VEPP-3 electron storage ring. The storage cell was found to increase the target thickness by approximately a factor of three and no loss in polarization was observed.
\end{abstract}

\section{INTRODUCTION}

The development of high flux sources of polarized hydrogen and deuterium is essential for the study of spin-dependent structure of the nucleon and nuclei. At Argonne, interest centers around the study of the tensor analyzing power in electron-deuteron scattering ${ }^{1}$ and the asymmetry ${ }^{2}$ in deep inelastic polarized electron scattering from a polarized nucleon.

With regard to the first experiment we have formed a collaboration with members of the Institute of Nuclear Physics at Novosibirsk in order to perform the experiment in the VEPP-3 electron storage ring. Here, the experiment is expected to proceed in three stages: (i) the use of a simple passive storage cell ${ }^{3}$ and a conventional atomic beam source to obtain a target thickness of $>6 \times 10^{11} \mathrm{~cm}^{-2}$; (ii) an active high-density storage cell with a conventional source to increase the target thickness to approximately $1.0 \times 10^{13}$ atoms $/ \mathrm{cm}^{2}$; and (iii) a laser-driven source and 
the active storage cell to increase the thickness to $\gtrsim 1 \times .0^{14}$ atoms $/ \mathrm{cm}^{2}$.

The second effort, to measure the spin-structure function of the proton and neutron, consists of a feasioility study to determine whether or not the experiment can be performed at the newly-constructed HERA ring at DESY. Based upon experience with the newly-developed laserdriven source and the experiment at Novosibirsk with a storage cell in the VEPP-3 ring, a figure-of-merit for a possible target at HERA will be presented.

In addition to these experiments, there is considerable interest in developing internal target programs at the MIT-Bates Laboratory, NIKHEF, EROS, IUCF and the PEF ring at SLAC. At present, the only ongoing program of nuclear physics with internal targets is at the VEPP-3 ring in Novosibirsk. A new ring, NEP, dedicated to nuclear physics is presently under construction at Novosibirsk.

The central issues in providing polarized targets of practical thickness $\left(>10^{14}\right.$ atoms $\left./ \mathrm{cm}^{2}\right)$ is the development of high-flux polarized sources and high-density windowless storage cells. At Argonne we have approached the first problem by recognizing that there are rather severe limitations to the conventional atomic beam source, ${ }^{4}$ namely the high density of gas atoms near the entrance to the sextupole, and by developing a new method ${ }^{5}$ based oi spin-exchange optical pumping. ${ }^{6}$ The flux of polarized atoms is limited by laser power and the efficiency of transfersing the polarization of photons from the laser to nuclei. Since a typical 1 watt laser in the visible region corresponds to $\sim 3 \times 10^{18}$ photons/s, an efficiency of $10 \%$ would represent a remarkable advance in polarized sources of $H$ and $D$. Presently, conventional atomic beam sources are limited to a total intensity of approximately $3 \times 10^{16}$ atoms $/ \mathrm{s}$.

The laser-driven polarized source is based on the principle of spin exchange optical pumping. In this method, photon angular momentum is transferred to an intermediate alkali atom, which, through spin exchange collisions, will polarize the electron of the hydrogen or deuterium atom. The nucleus becomes polarized, since at low magnetic fields, the electronic and nuclear spins are coupled by the hyperfine interaction. Compared to conventional sources the key advantages for internal target applications are: (i) the flux from the source is expected to be limited only by laser power; (ii) the source emittance is easily tailored to meet storage cell requirements; (iii) low-background gas load to the storage ring.

A closely related problem is the efficiency with which a source can in sct polarized atoms into a storage cell. The feasibility study of the use of storage cells for atoms in a storage ring is presently being conducted by the Argonne-Novosibirsk collaboration and the progress, thus far, is reported by D. Toporkov ${ }^{7}$ at this conference. 


\section{EXPERIMENTAL}

Figure 1 shows the prototype laser-driven polarized hydrogen deuterium source. An $\mathrm{Ar}^{+}$-pumped standing wave dye laser operating with Pyridine 2 dye provides the $770 \mathrm{~nm}$ radiation required to optically pump the D1 line of potassium (K). The spectral density of the laser is tailorcd to match the doppler-broadened absorption profile of the $K$ vapor $(\simeq 1.5 \mathrm{GHz}$ ) using an electro-optic modulator (EOM). The "tailored" laser output is sent through a linear polarizer and circular polarizer and then into the spin exchange cell.

The spin exchange cell is constructed of pyrex as an integral unit with the rf dissociator ( $H / D$ source) and the $K$ reservoir. The cell interior is coated with a polarization preserving material, drifilm. ${ }^{8}$ The cell is placed in a static field of $\sim 10 \mathrm{G}$. The flux (density) of the $\mathrm{H} / \mathrm{D}$ atoms is controlled by a mass flowmeter in conjunction with a servodriven needle-valve. The density of the $K$ atoms is independently controlled through the reservoir temperature. The entire cell is enclosed in a copper oven and heated to $\sim 230^{\circ} \mathrm{C}$ to prevent alkali condensation. Typical $\mathrm{K} / \mathrm{H}$ density ratios are $1-3 \%$.

The usable flux of $H / D$ atoms is determined through a combination of measurements. First, the total flow of $\mathrm{H}_{2} / \mathrm{D}_{2}$ is measured at the input of the rf dissociator as described above. Since the entire flow through the of dissociator is potentially useful (excluding losses through recombination, chemical reaction etc.), it is only necessary to measure, at the output, the fraction of $\mathrm{H}_{2} / \mathrm{D}_{2}$ which rewains dissociated. This is done using a mechanical chopper and quadrupole mass spectrometer (QMS) in combination with a lock-in amplifier. The molecular $\left(\mathrm{H}_{2}\right.$ or $\left.\mathrm{D}_{2}\right)$ intensity is measured with the if to the dissociator first turned off and then on. The fraction dissociated is simply: DF=1-I (rf on)/I(rf off). A typical fraction $f(C r$ a 1000 bounce cell with a well-prepared drifilm surface is $70-80 \%$.

The other important parameter in source characterization is the polarization of the $\mathrm{H}$ atoms. This is measured using an opticallydetected magnetic resonance technique. ${ }^{9}$ In this method, the transparency of the $\mathrm{K}+\mathrm{H}$ sample to the resonance radiation is decreased by including Zeeman (depolarizing) transitions with a small coil placed at right angles to the holding field. The decreased ransparency causes an increase in resonance fluorescence which is detected by a photodiode as shown in Fig. 1. Both the polarization of the $K$ and the $H / D$ can be detected in this manner by sweeping the depolarizing rf field over the appropriate frequency range. The depolarizing field is amplitude modulated and the detected fluorescence is processed through a lock-in amplifier in order to reduce noise resulting from laser intensity fluctuations. 


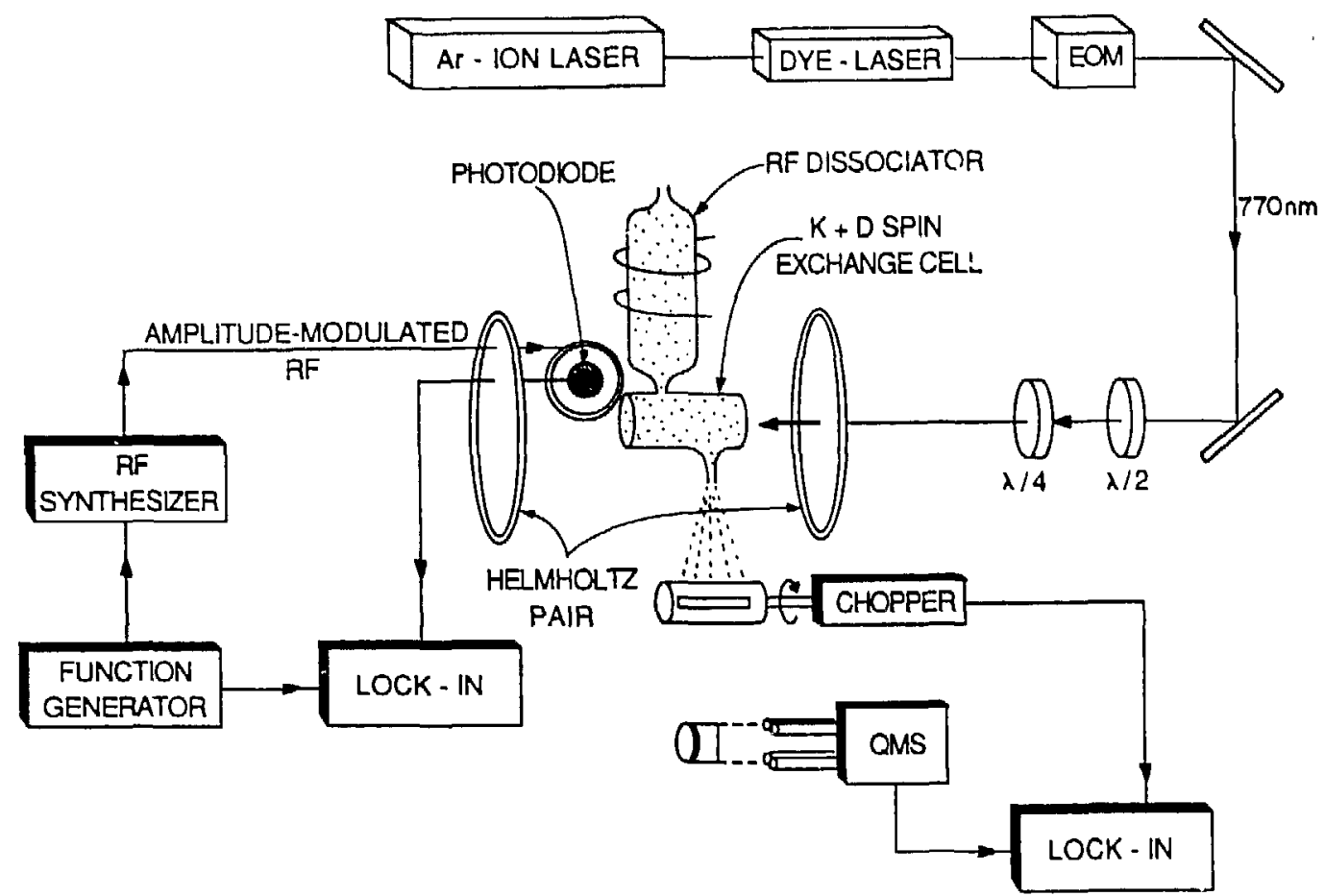

Fig. 1. Schematic diagram of the laser driven polarized source of $H / D$.
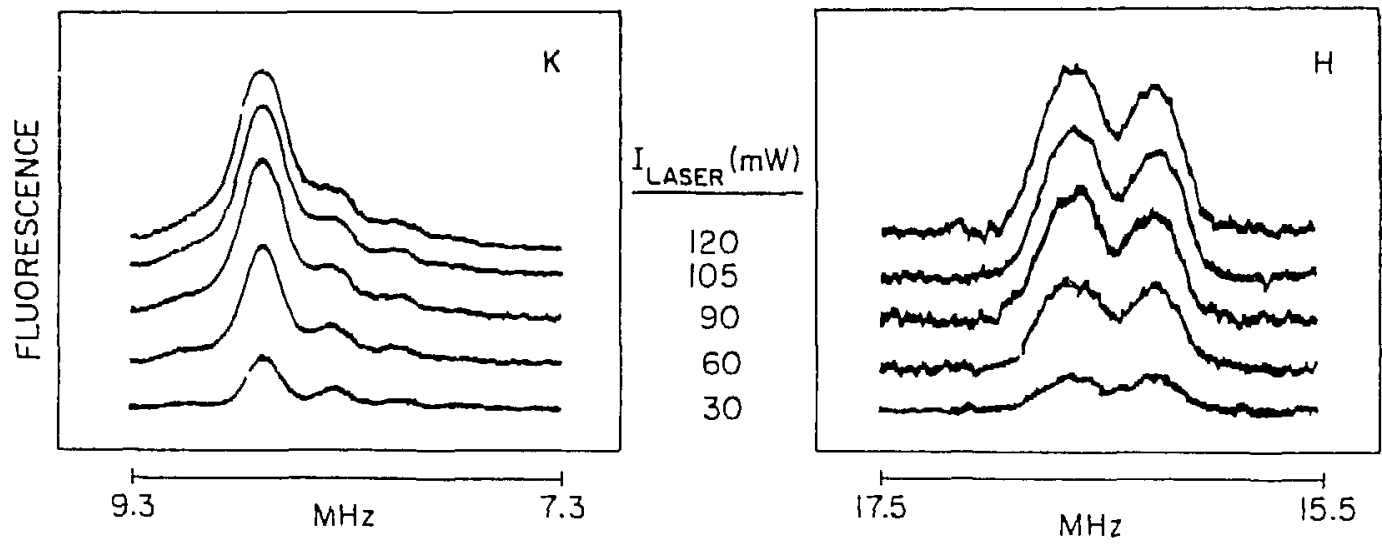

Fig. 2. Zeeman transition signals for $K$ (left) and $H$ (right) as a function of incident laser power. 
Figure 2 shows a typical family of Zeeman signals for $K$ and $H$ as a function of laser power. From these curves it is possible to determine the polarization of the $K$ and $H$ vapor within the spin exchange cell. The populations of the magnetic sublevels of both $K$ and $H$ are assumed to be in spin equilibrium, i.e. describable by a spin temperature, $\beta$. In this case, the relative state populations $\left(\mathrm{n}_{\mathrm{i}}=\mathrm{Ce}^{\mathrm{m}_{\mathrm{i}} \beta}\right.$ ) can be deduced by the Zeeman transition signal strength which is proportional to $|(F, m|J,| F, m+1)|^{2} g(\nu) \Delta n$. The top trace for $K$ shows a vector polarization of $82 \%$, whereas the corresponding trace for $H$ shows a polarization of only $15 \%$.

A similar trace for polarized deuterium in the spin exchange cell is shown in Fig. 3. Here, one expects to see in general four Zeeman transitions, however, at this small magnetic field the $2 \rightarrow 3$ and $5+6$ transitions are indistinguishable and show up at the center peak in the figure. The high quality of these data is indicative of an improvement in the uniformity of the magnetic field from the Helmholtz pair as compared with the traces in Fig. 2. The polarization of the deuterons in this figure is determined to be $25 \%$ at a flow rate of $6 \times 10^{16}$ atoms $/ \mathrm{s}$. This polarization includes the measured fractions of atoms to molecules emerging from the cell.

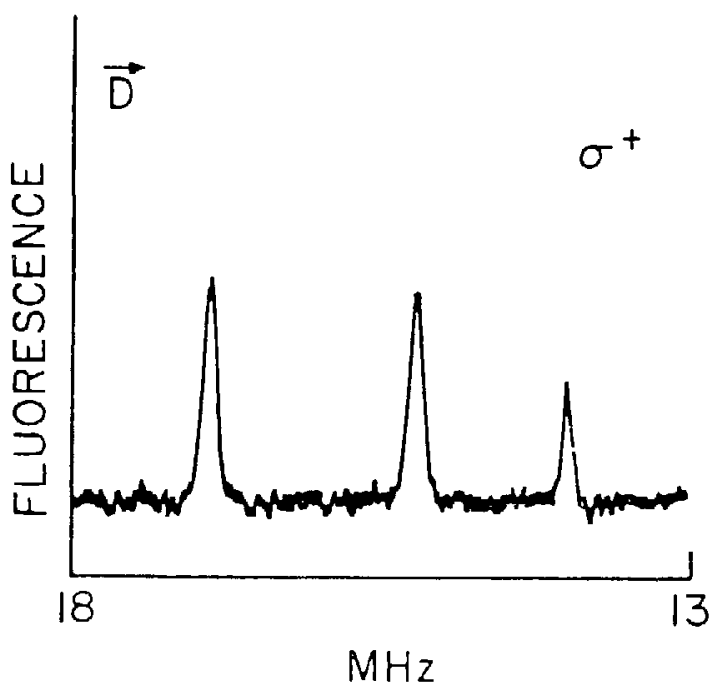

Fig. 3. Observed Zeeman signal for polarized deuterium in the spin-exchange cell.

\section{DISCLAIMER}

This report was prepared as an account or work sponsored by an agency of the United States Government Nether the IInited States Governmen' nor any agency thireof, nor any of their employees, makes any warranty, express or implied, or assumes any legal liahility or responsibility for the accuracy. completeness, or usefulness of any information, apparatus, product, or process disclused, or represents that its use would not infringe privately owned rights. Reference herein $u$ any specific commercial product, process, or service by trade name, trademark. manufacturet. or atherwise dies not necessarily constitule or imply ths endorsement, recommendation, or favoring hy the IInted sitates ciovernment or any agency thereof. The views and opinums of authurs expressed heresn do not necessarsly state ur reflect thuse of the Unted States Ciovernment or any agency thereof 


\section{RESULTS AND DISCUSSION}

The best performance of the prototype source that we so far have achieved is a flux of $8 \times 10^{16} \mathrm{H}$ atoms/s, with a polarization of $30 \%$ and a dissociation fraction of $80 \%$ through a $4 \mathrm{~mm}$ diameter spout. Comparable results are cbtained for deuterium. This is within a factor of 5 of the benchmark conventional atomic beam source at Bonn ${ }^{4}$ (flux $\sim 3 \times 10^{16}$ atoms/s, polarization $\sim 90 \%$, through a $\sim 7 \mathrm{~mm}$ diameter opening). It is, however, much lower than the design goal of $4 \times 10^{17}$ atoms/s with a vector polarization of $50 \%$.

The various factors limiting source performance are inter-related. The flux can be easily increased. The polarization can not. The H/D flux is increased by enlarging the inlet hole from the of dissociator to the spin-exchange cell. The polarization is enhanced by increasing the spin-exchange rate relative to the depolarization rate. The spin-exchange rate is proportional to the $\mathrm{K}$ density, which cannot be arbitrarily increased due to radiation trapping. ${ }^{10}$ The depolarization rate is dominated by collisions with the walls. Our data show that the depolarization probability per wall collision hor hydrogen atoms is $\sim 1 / 300$. This is significantly poorer than previously reported results for hydrocarbon surfaces. A Monte Carlo analysis indicates that an improvement by a factor of 1.8 in polarization can be expected by further optimization of the spin-exchange cell geometry.

\section{PASSIVE STORAGE CELL IN THE VEPP-3 RING}

A storage cell in the form of an A $\ell$ elliptical tube of dimensions $24 \times 46 \times 940 \mathrm{~mm}$ was installed in the VEPP-3 storage ring. A cylindrical tube of dimensions $10 \mathrm{~mm}$ diameter and $60 \mathrm{~mm}$ long provided the inlet for the polarized atoms from the Novosibirsk atomic beam source. The location of this cell in the storage ring is shown schematically in Fig. 4. Drifilm was selected as the coating because of its excellent vacuum properties, high temperature stability, spin-relaxation characteristics and low hydrogen recombination coefficient. The large cross-sectional size of the tube was based upon the large electron beam size during the filling procedure of the VEPP-3 ring. This large size severely limits the density of the stored atoms. The purpose of the phase I storage cell test was to study the feasibility of a stcrage cell in an electron ring under the simplest possible circumstances before proceeding with an active cell in phase II.

A relatively large magnetic guide field was applied to the entire storage volume of the cell. A transverse field was applied to the central $10 \mathrm{~cm}$ and solenoidal fields to the remainder of the tube as indicated in Fig. 4. This guide field was necessary to compensate for the time-varying magnetic fields of the electron beam pulses. During the 


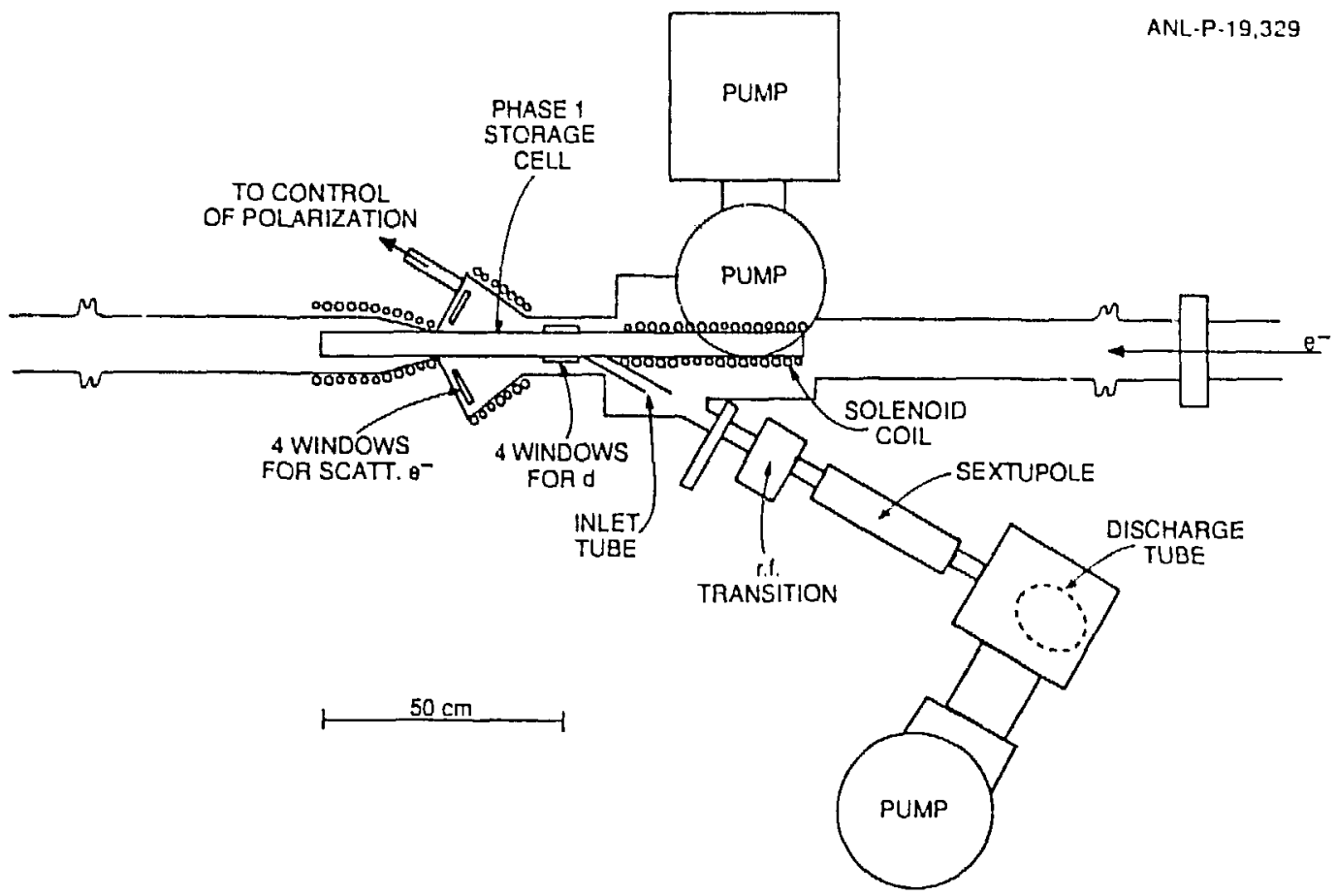

Fig. 4. Schematic diagram of the storage cell $i_{11}$ the VEPP-3 ring.

course of the tests the guide field was increased in stages as more solenoids were added. The effect of the increase in the static magnetic field and the electron beam pulse width is indicated in Table I. The entry, $a$, in the last column of the table is the asymmetry for the $\vec{D}(e, p n) e^{\prime}$ reaction. For the case of the jet, this asymmetry was found to be $0.235 \pm 0.026$. The first entry in the table shows that for high current $(200 \mathrm{~mA})$ short pulses and a low guide field, the asymmetry observed with the storage cell is small. As a test of whether this depolarization is due to collisions with the walls of the storage cell or from the magnetic field of the electron beam. The electron beam pulse width was increased to a width of 8 ns and 2 pulses were circulated in the ring to give an average current of $200 \mathrm{~mA}$. With these conditions the asymmetry was found to increase to $0.19 \pm 0.04$ and was consistent with the jet data. This meant that the depolarization was not due to a problem with the drifilm wall coating. When the guide magnetic field was increased so that the minimum field in the cell was approximately $350 \mathrm{G}$ a large asymmetry was observed for the short-pulse mode of 
VEPP-3 as shown by the last entry in the table. In this case $w$ : achieved a highly polarized target of deuterons in a storage cell in the VEPP-3 ring. The increase in target thickness over the jet target was found to be about a factor of three in this simple storage cell. With the active phase II cell a factor of approximately 25 in target thickness is expected and is discussed in the following section.

Table I

Observed asymmetries for the $\bar{D}(e, p n) e^{\prime}$ reaction as a function of electron beam current and pulse width and the magnetic guide field.

\begin{tabular}{cccccc}
\hline $\begin{array}{l}\text { Current } \\
\text { Pulse } \\
(\mathrm{mA})\end{array}$ & $\begin{array}{c}\text { Number } \\
\text { Pulses } \\
(\mathrm{ns})\end{array}$ & $\begin{array}{c}\text { Pulse } \\
\text { Width }\end{array}$ & $\begin{array}{c}\text { Magnetic } \\
\text { Field } \\
\text { Stage }\end{array}$ & $\mathrm{E}_{\mathbf{e}}$ & a \\
$(\mathrm{GeV})$ & \\
\hline & & & & & \\
200 & 1 & 1 & 1 & 2.0 & $0.053 \pm 0.021$ \\
100 & 2 & 8 & 2 & 0.56 & $0.19 \pm 0.04$ \\
60 & 2 & 1 & 3 & 2.0 & $0.28 \pm 0.06$ \\
\hline
\end{tabular}

\section{FIGURE-OF-MERIT FOR INTERNAL POLARIZED TARGET}

Based upon the experience with an internal polarized target consisting of a storage cell injected by a conventional polarized source in the VEPP-3 ring and the plans for phases II and III of these studies, relative figures of merit for polarized target configurations may be given. If we define the figure-of-merit as target thickness $\times$ (polarization) ${ }^{2}$, i.e. $\mathrm{np}_{\mathrm{z}}^{2}$, then the figure-of-merit for various target configurations is summarized in Table II. The first two entries in the table are based upon experience at Novosibirsk for the jet target and the passive storage cell configuration. The third entry is the figure-of-merit that is expected in the phase II experiment with the Argonne active storage cell and the Novosibirsk atomic beam source. Note that this figure-of-merit is an improvement by a factor of 25 compared with the jet target. The fourth entry in the table indicates the improvement expected if the active storage cell is used in conjunction with the achieved results for 
the laser-driven source. This would represent only a factor of 2 increase over the conventional method and the laser-driven source would have to be developed further to represent a clear advantage. For reascnable projections of improvement in the laser-driven source, one would expect an improvement by a factor of approximately 30 over the conventional scheme as indicated by the last entry in the table.

Table II

Achieved figures of merit for a polarized jet target and the phase I storage cell. Extrapolated figures of merit for phases II and III.

\begin{tabular}{|c|c|c|c|c|}
\hline Condition & $\begin{array}{l}\text { Useful } \\
\text { Jet Flux } \\
\left(\mathrm{s}^{-1}\right)\end{array}$ & $\left(\times 10^{12}\right.$ & $\begin{array}{r}P_{1} \\
\left.c m^{-2}\right)\end{array}$ & $\begin{array}{c}n p_{x}^{2} \\
\left(\times 10^{12} \mathrm{~cm}^{-2}\right)\end{array}$ \\
\hline Jet & $2 \times 10^{16}$ & 0.2 & 0.8 & 0.13 \\
\hline $\begin{array}{l}\text { Phase I } \\
\text { Cell }\end{array}$ & $4 \times 10^{15}$ & 0.6 & 0.9 & 0.49 \\
\hline $\begin{array}{l}\text { Phase II } \\
\text { Cell }\end{array}$ & $4 \times 10^{15}$ & 3.9 & 0.9 & 3.2 \\
\hline $\begin{array}{l}\text { Phase II } \\
\text { Cell + Laser } \\
\text { Driven Source }\end{array}$ & $8 \times 10^{16}$ & 100.0 & 0.24 & 6.0 \\
\hline $\begin{array}{l}\text { Phase III } \equiv \\
\text { (Phase II Cell } \\
+ \text { Projected } \\
\text { Laser Source } \\
\text { Improvement) }\end{array}$ & $4 \times 10^{17}$ & 510 & 0.44 & 100 \\
\hline
\end{tabular}

This is the initial demonstration of a laser-driven polarized source of hydrogen and deuterium. While not yet up to design goals, the results are encouraging. We expect to be able to improve source performance by: (i) minimizing radiation trapping by careful cell design and alkali selection; (ii) developing better surface coatings; and (iii) increasing laser power.

The first use of a storage cell for polarized atoms in an electron storage ring was demonstrated to be successful. The target thickness 
was increased by a factor of 3 larger than that of the atomic jet and no loss in polarization was observed. A phase II storage cell is under construction and a factor of 25 improvement is expected.

\section{ACKNOWLEDGEMENTS}

We would like to thank J. Gregar for state-of-the-art glassblowing, G. Thomas for assistance in surface coatings, and J. Berkowitz and the late L. Goodman for helpful discussions.

This research is supported in part by the U. S. Department of Energy, Nuclear Physics Division, under contract W-31-109-ENG-38.

\section{REFERENCES}

1. R. J. Holt et al., Nucl. Phys. A446, 389c (1985); Proceedings of the 1st Conference on Intersections Between Particle and Nuclear Physics, AIP No. 123, 499 (1984).

2. D. H. Beck et al., Proceedings of the 3rd Conference on Intersections Between Particle and Nuclear Physics, TRIUMF, Rockport, ME, May 14-19, 1988.

3. M. D. Barker et al., A target of polarized hydrogen by storage of atoms in a coated pyrex vessel, in: Polartzation Phenomena in Nuclear Physics, ed. G. G. Ohlsen at al. (AIP 1981) pp 931-932.

4. H. G. Mathews et al., Nucl. Instr. and Meth. 213, 155 (1983).

5. R. J. Holt, Proceedings of the Workshop on Polarized Targets in Storage Rings, ANL Report No. 84-50 (1984), p 103; L. Young et al., Nucl. Instr. and Meth. B24/25, 963 (1987).

6. H. G. Dehmelt, Phys. Rev. 109, 381 (1958).

7. S. I. Mishnev et al., in these Conference Proceedings.

8. D. R. Swenson and L. W. Anderson, Nucl. Instr. and Meth. B28, 627 (1988); M. A. Bouchiat and J. Brossel, Phys. Rev. 147, 41 (1966).

9. W. E. Bell and A. L. Bloom, Phys. Rev. 107, 1559 (1957); R. Knize and J. Cecchi, Phys. Lett. 113A, 255 (1985).

10. D. Tupa and L. W. Anderson, Phys. Rev. $\underline{A 36}, 2142$ (1987). 